Research Article

\title{
Additional value of a combined genetic risk score to standard cardiovascular stratification
}

Andreia Pereira $^{1,2}$, Maria Isabel Mendonca ${ }^{1}$, Sofia Borges ${ }^{1}$, Ana Célia Sousa ${ }^{1,2}$, Sónia Freitas ${ }^{1}$, Eva Henriques $^{1}$, Mariana Rodrigues ${ }^{1}$, Ana Isabel Freitas ${ }^{1,3}$, Graça Guerra ${ }^{1,3}$, Carolina Freitas ${ }^{1}$, Décio Pereira ${ }^{1}$, António Brehm ${ }^{3}$ and Roberto Palma Dos Reis ${ }^{2}$

${ }^{1}$ Unidade de Investigação, Hospital Dr. Nélio Mendonça, Funchal, Portugal.

${ }^{2}$ Faculdade de Ciências Médicas, Universidade Nova de Lisboa, Lisboa-Portugal.

${ }^{3}$ Laboratório de Genética Humana, Universidade da Madeira, Campus Universitário da Penteada, Madeira, Portugal.

\begin{abstract}
The utility of genetic risk scores (GRS) as independent risk predictors remains inconclusive. Here, we evaluate the additive value of a multi-locus GRS to the Framingham risk score (FRS) in coronary artery disease (CAD) risk prediction. A total of 2888 individuals ( 1566 coronary patients and 1322 controls) were divided into three subgroups according to FRS. Multiplicative GRS was determined for 32 genetic variants associated to CAD. Logistic Regression and Area Under the Curve (AUC) were determined first, using the TRF for each FRS subgroup, and secondly, adding GRS. Different models (TRF, TRF+GRS) were used to classify the subjects into risk categories for the FRS 10-year predicted risk. The improvement offered by GRS was expressed as Net Reclassification Index and Integrated Discrimination Improvement. Multivariate analysis showed that GRS was an independent predictor for CAD $(O R=1.87$; $p<0.0001)$. Diabetes, arterial hypertension, dyslipidemia and smoking status were also independent CAD predictors $(p<0.05)$. GRS added predictive value to TRF across all risk subgroups. NRI showed a significant improvement in all categories. In conclusion, GRS provided a better incremental value in intermediate subgroup. In this subgroup, inclusion of genotyping may be considered to better stratify cardiovascular risk.
\end{abstract}

Keywords: Coronary artery disease, genetic risk score, Framingham score, risk prediction, risk factors.

Received: June 6, 2017; Accepted: January 29, 2018.

\section{Introduction}

The most important Traditional Risk factors (TRF) for Coronary Artery Disease (CAD) include dyslipidemia, arterial hypertension, diabetes, obesity, smoking, lack of physical activity and stress (Chan and Boerwinkle, 1994). However, some patients can develop vascular disease without conventional risk factors.

Although the familial nature of CAD has been documented for many years (Marenberg et al., 1994; LloydJones et al., 2004; Murabito et al., 2005) and the addition of family history has been shown to improve risk prediction (Ridker et al., 2007; Ridker et al., 2008), the genetic variants responsible for the increased familial risk were, until recently, unknown. Genome-Wide Association Studies (GWAS) have uncovered several common genetic variants (single nucleotide polymorphisms, or SNPs) that are robustly associated with CAD and have been replicated in

Send correspondence to Andreia Micaela Pereira. Unidade de Investigação, Hospital Dr. Nélio Mendonça, Avenida Luís de Camões, $\mathrm{n}^{-}$57, 9004-514 Funchal, Portugal. E-mail: andreiapereira21@gmail.com multiple independent samples (Welter et al., 2014). The identification of these genetic variants provides an opportunity to evaluate whether addition of a genetic risk score (GRS) to risk models may improve predictive power (Ganna et al., 2013).

There is an increasing interest in the potential use of GRS in cardiovascular disease, because this could increase the number of preventive and therapeutic interventions in individuals and groups with high genetic risk that are not obvious candidates to these interventions using the current standard stratification. Usual cardiovascular risk stratification uses family history, TRF evaluation, and is quantified into scores like Framingham risk score and EuroSCORE (Pencina et al., 2011). However, due to the potential financial and medical costs associated with measuring these new markers like GRS, their ability in improving the prediction of $\mathrm{CAD}$ outcomes over existing risk models needs to be rigorously accessed. Effective statistical tools for evaluating the incremental value of the novel markers over the routine clinical risk factors are crucial in the field of outcome prediction. 
Here, we evaluate whether a GRS, based on 33 SNPs associated to $\mathrm{CAD}$, is independent of the vascular risk explainable by conventional risk factors and can improve the predictive capacity of TRF in the assessment of CAD risk, according to FRS subgroups (low risk $<10 \%$, intermediate risk $10-20 \%$ or high risk $>20 \%$ ). Using the Net Reclassification Index (NRI) and Integrated Discrimination Index (IDI), we investigate the performance of combined stratification including TRF and GRS in CAD risk assessment.

\section{Subjects and Methods}

\section{Study population}

A case-control study was performed with 2888 individuals (mean age of 53.0 \pm 7.9 years), including 1566 consecutive coronary patients and 1322 controls. These were divided into three subgroups according to FRS: low risk (FRS $<10, \mathrm{n}=1312,68.7 \%$ male), intermediate risk $(10 \leq \mathrm{FRS} \leq 20, \mathrm{n}=1049,83.1 \%$ male $)$ and high risk (FRS $>20, \mathrm{n}=527,90.1 \%$ male). Cases and controls were matched for age and gender.

Determination of CAD was adjucated by a dedicated intervention cardiologist. Angiographically proven CAD was considered significant if $\geq 1$ coronary lesions of $\geq 70 \%$ stenosis in $\geq 1$ major coronary artery or its primary branches. Absent or non-flow limiting CAD was excluded from this study. The control group consisted of healthy volunteers selected from the same population with no symptoms or history of CAD. All controls underwent clinical and phenotype assessment of TRF, an electrocardiogram, and, if needed, complementary exercises stress tests or a Stress Echocardiography. Population stratification analysis was performed in our population set to account for possible genetic admixture and no significant genetic outliers $(<5 \%)$ were identified with Principal Component Analysis (PCA) (Abdi and Williams, 2010).

The study was conducted according to the Declaration of Helsinki, the protocol was reviewed and approved by the Hospital ethics committee, and all patients provided written informed consent.

\section{Data collection}

Data was collected from all subjects in a standardized file comprising demographic, clinical characteristics and TRF (gender, age, level of exercise, smoking status, arterial hypertension, dyslipidemia, diabetes, and family history of CAD, body mass index (BMI), heart rate and pulse wave velocity (PWV). 'Smoking status' refers to current smokers or subjects with less than 5 years of smoking cessation (Mons et al., 2015).

Arterial Hypertension was considered when patients, at the entry into this study, were already diagnosed and/or had been on antihypertensive medication for more than 3 months, or newly diagnosed hypertensives with systolic blood pressure (SBP)/diastolic blood pressure (DBP) $\geq 140 / 90 \mathrm{mmHg}$ measured on at least 3 occasions (Chobanian et al., 2003).

Subjects with LDL $>100 \mathrm{mg} / \mathrm{dL}, \mathrm{HDL}<40 \mathrm{mg} / \mathrm{dL}$ for men, and $<45 \mathrm{mg} / \mathrm{dL}$ for women, non HDL (Total cholesterol - HDL) $>130 \mathrm{mg} / \mathrm{dL}$, triglycerides $>150 \mathrm{mg} / \mathrm{dL}$ or Apo $\mathrm{B}>100 \mathrm{mg} / \mathrm{dL}$ were classified as having dyslipidemia (Expert Panel on Detection Evaluation and Treatment of High Blood Cholesterol in adults, 2001). Subjects were classified as having diabetes if taking oral anti-diabetic medication or insulin or if fasting plasma glucose was higher than $7.0 \mathrm{mmol} / 1$ or $126 \mathrm{mg} / \mathrm{dL}$ (Genuth et al., 2003).

Family history of CAD was considered if a female relative (mother or sister) had presumed CAD disease before 65 years, or a male relative (father or brother) had CAD disease before 55 years old. The definition of other traditional risk factors was based on standard criteria, as previously reported (Asmar et al., 1995; National Institute of Health, National Heart, Lung, and Blood Institute North American Association for the Study of Obesity, 2000).

\section{Biochemical analysis}

Blood samples were extracted after 14-16 hours fasting. Biochemical analyses were performed at the Central Laboratory of the Hospital, according to standard techniques. For measurement of total cholesterol, HDL, LDL, triglycerides and glucose, blood samples were placed in dry tubes, centrifuged half an hour late at $3500 \mathrm{xg}$ and subsequently quantified by an enzymatic technique using an $\mathrm{AU}$ 5400 autoanalyzer (Beckman Coulter). Biochemical markers such as lipoprotein-a ( $L p(a)$ ), apolipoprotein B (Apo B), and high sensitivity $\mathrm{C}$-Reactive Protein (hs-CRP) were quantified by Immunoturbidimetry using an AU 5400 automatic system (Beckman Coulter).

\section{SNP selection}

SNPs were selected either from GWAS or candidate gene association studies (Coronary Artery Disease Consortium, 2011; Schunkert et al., 2011). These SNPs were either previously tested in a sample of our population, or in a genetically similar southern European cohort. Entering criteria included genes described in previous studies with an Odds Ratio (OR) for $\mathrm{CAD} \geq 1.1$ and, simultaneously, with a Minor Allele Frequency (MAF) $>5 \%$. Genes with low Hardy-Weinberg equilibrium $\mathrm{p}<0.002$ (after Bonferroni correction) were excluded.

According to their possible CAD-related function, we have included 32 genes associated to cell cycle, cellular migration and inflammation (rs1333049 (9p21.3), rs4977574 (CDKN2B), rs618675 (GJA4), rs17228212 (SMAD3), rs17465637 (MIA3), rs12190287 (TCF21), rs3825807 (ADAMTS7), rs11556924 (ZC3HC1), rs12526453 (PHACTR1); genes involved in pro-oxidative status (rs1801133 (MTHFR 677), rs1801131 (MTHFR 1298), rs705379 (PON 1), rs662 (PON192), rs854560 (PON 55), rs6922269 (MTHFD1L); genes associated with modifiable 
risk factors such as lipids metabolism, hypertension and diabetes/obesity (rs2114580 (PCSK9), rs20455 (KIF6), rs7412/rs429358 (APOE), rs964184 (ZNF259), rs599839 (PSRC1), rs5186 (AT1R), rs699 (AGT), rs4340 (ACE), rs4402960 (IGF2BP2), rs1326634 (SLC30A8), rs266729 (ADIPOQ), rs7903146 (TCF7L2), rs17782313 (MC4R), rs1801282 (PPARG), rs1884613 (HNF4A), rs8050136 (FTO) and rs1376251 (TAS2R 50).

Further SNP inclusion was not performed at this time due to low expectation of improvement in overall risk reclassification and cost effectiveness limitations.

Details of SNPs used in GRS model are presented in Supplementary Table S1.

\section{Genotype analysis}

Genetic analysis was performed at the Human Genetics Laboratory of the University of Madeira. Genomic DNA was extracted from $80 \mu \mathrm{L}$ of peripheral blood using a standard phenol-chloroform method. A TaqMan allelic discrimination assay for genotyping was performed using labeled probes and primers pre-established by the supplier (TaqMan SNP Genotyping Assays, Applied Biosystems). All reactions were done on an Applied Biosystems 7300 Real Time PCR System and genotypes were determined using the 7300 System SDS Software (Applied Biosystems) without any prior knowledge of the individual's clinical data. Quality check of genotyping techniques was maintained by the inclusion of one non-template control (NTC) in each plate of 96 wells. All SNPs TaqMan assays had blind duplicates accounting for $20 \%$ of all samples. Some SNP genotypes were randomly confirmed by conventional direct DNA sequencing, as $10-15 \%$ of all samples were re-amplified for sequencing.

\section{Statistical analysis}

Deviation from Hardy-Weinberg equilibrium for the 33 genotypes at individual loci were assessed using the Chi-square test and $p<0.002$ with Bonferroni correction for all SNPs included. LPA gene variant was excluded for further analyses due to its low Hardy-Weinberg equilibrium $(p<0.002)$.

Comparisons of baseline characteristics and biochemical data of cases and controls were analyzed by Chisquare tests for categorical variables, and Student's $t$-test or Mann- Whitney tests were performed for continuous variables, as appropriate. Genotypic frequencies were determined from observed counts and compared by Chi-square analysis.

To evaluate the impact of genotype frequencies and Odds Ratios (OR) on the overall discriminative accuracy of genetic risk models, we assessed the AUC. SNPs associated with $p$-values less than or equal to 0.05 entered the race-specific GRS with a coding value of 2 for the mutated genotype (risk), 1 for the heterozygote, and 0 for the homozygous wild-type genotype. The GRS was constructed un- der a multiplicative model (multiplying the odds ratio of each genotype for all the 32 genetic variants). The maximum number of risk alleles possessed by one individual was 36 and the minimum was 13 (mean \pm standard deviation $(\mathrm{SD})=23.96 \pm 3.63)$. The increasing loci were assumed to have an additive effect, and the GRS was normally distributed.

ROC curves based on the GRS + Framingham model were compared to a Framingham model alone (Figure 1). For each risk subgroup, the GRS was included in a ROC model containing the TRF. A new combined risk score was calculated for each individual (TRF + GRS) by multiplying each model coefficient by the associated risk variable and then summing the products. The AUC equals the probability that a classifier will rank a randomly chosen positive instance higher than a randomly chosen negative one. ROC curve analysis was performed using the MedCalc software. Non-parametric methods developed by DeLong et al. (1988) were used to test for significant differences between ROC curves.

NRI was computed according to the continuous method and applied to the case- control studies (Pencina et al., 2011). We further calculated categorical NRI for each Framingham subgroup. In each risk group the number of individuals reclassified intohigher and lower risk categories is presented (Supplementary Table S2). NRI was defined as the percentage of subjects changing categories in each subgroup when adding the new markers and IDI as the improvement of the difference in average of predicted probabilities between cases and controls. Both measures

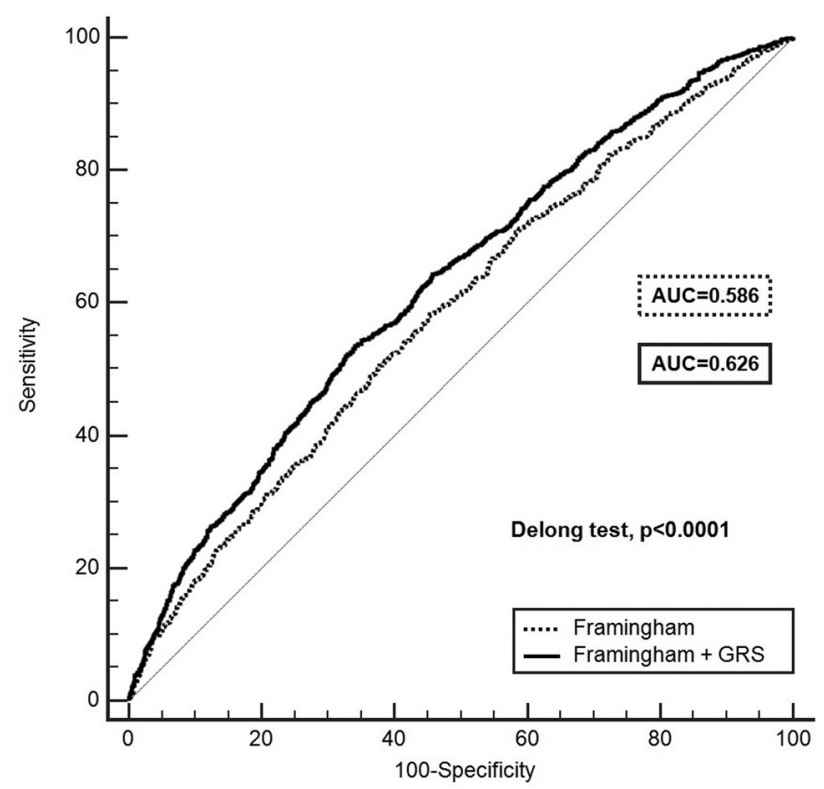

Figure 1 - ROC curves based on the GRS + Framingham model compard to Framingham model alone. The two curves are based on logistic regression models incorporating Framingham risk with and without GRS. AUC indicates area under curve. The DeLong test compares the difference between the two AUCs and showed statistical significance. 
were obtained with the PredictABEL package available in $\mathrm{R}$ software (version 3.2.0). Discriminative capacity (AUC) was accessed for evaluation of specificity and sensitivity with MedCalc version 13.3.3.0, and other statistics were analyzed by the Statistical Package for the Social Sciences, SAS Software version 19.0). All $p$-values were two-sided, and statistically significant for $p<0.05$.

\section{Results}

Table 1 compares baseline characteristics between $\mathrm{CAD}$ and control groups. According to the selection criteria, age and gender did not differ significantly between the two groups. In contrast, CAD patients were more likely to have a higher prevalence of modifiable risk factors, such as dyslipidemia, tobacco use, hypertension, and diabetes $(p<0.05)$. Also BMI, PWV, family history of CAD and lower level of exercise were significantly more frequent among cases $(p<0.05)$. Lower heart rates among patients potentially reflect a better heart rate control $(p<0.0001)$. Across all Framingham score subgroups, the GRS was significantly lower in controls that CAD patients $(p<0.0001)$.

A logistic regression under a Forward Wald method was performed including GRS and TRF (Table 2). In this multivariable analysis, GRS was an independent predictor for $\mathrm{CAD}(\mathrm{OR}=1.87 ; 95 \% \mathrm{CI}: 1.58-2.21)$, with statistical significance $(\mathrm{p}<0.0001)$. Even after a Bonferroni Correction, GRS remained significant at a $p<0.01(0.05 / 5)$ level. Furthermore, smoking status $(\mathrm{OR}=3.44 ; 95 \% \mathrm{CI}$ : $2.89-$ 4.10); diabetes $(\mathrm{OR}=3.19$; $95 \% \mathrm{CI}: 2.61-3.91)$; arterial hypertension $(\mathrm{OR}=2.10 ; 95 \% \mathrm{CI}: 1.77-2.48)$ and dyslipi-

Table 1 - Baseline characteristics for both CAD patients and controls.

\begin{tabular}{|c|c|c|c|c|}
\hline Variables & $\begin{array}{c}\text { Total } \\
(\mathrm{n}=2888)\end{array}$ & $\begin{array}{c}\text { Cases } \\
(\mathrm{n}=1566)\end{array}$ & $\begin{array}{l}\text { Controls } \\
(\mathrm{n}=1322)\end{array}$ & $p$-value \\
\hline Male sex, $n(\%)$ & $2248(77.8)$ & $1238(79.1)$ & $1010(76.4)$ & 0.087 \\
\hline Age, years & $53 \pm 7.9$ & $53.3 \pm 8$ & $52.7 \pm 7.8$ & 0.053 \\
\hline Dyslipidemia $^{\dagger}, n(\%)$ & $2344(81.2)$ & $1414(90.3)$ & $930(70.3)$ & $<0.0001$ \\
\hline Total Cholesterol, mg/dl & $193(77-437)$ & $180(77-437)$ & $205(92-360)$ & $<0.0001$ \\
\hline $\mathrm{HDL}, \mathrm{mg} / \mathrm{dl}$ & $44(12-116)$ & $41(18.2-115.8)$ & $48(12-116)$ & $<0.0001$ \\
\hline $\mathrm{LDL}, \mathrm{mg} / \mathrm{dl}$ & $114.8(9.6-298)$ & $104.6(15.6-298)$ & $127.2(9.6-251)$ & $<0.0001$ \\
\hline Lipoprotein (a), mg/dl & $15.1(0.5-241)$ & $20.4(0.5-241)$ & $12.8(0.6-236)$ & $<0.0001$ \\
\hline Apolipoprotein B, mg/dl & $93.2(2.9-256.9)$ & $93.9(4.9-256.9)$ & $92.5(2.9-212.7)$ & $<0.0001$ \\
\hline Triglycerides, mg/dl & $132(30-2500)$ & $141(31-2500)$ & $121(30-1361)$ & $<0.0001$ \\
\hline Smoking status, $n(\%)$ & $1039(36)$ & $730(46.6)$ & $309(23.4)$ & $<0.0001$ \\
\hline Hypertension, $n(\%)$ & $1814(62.8)$ & $1114(71.1)$ & $700(53)$ & $<0.0001$ \\
\hline $\mathrm{SBP}, \mathrm{mmHg}$ & $137.1 \pm 19.6$ & $137.9 \pm 20.8$ & $136.2 \pm 18.1$ & 0.024 \\
\hline DBP, $\mathrm{mmHg}$ & $83.2 \pm 11.5$ & $82.6 \pm 11.8$ & $83.9 \pm 11.1$ & 0.002 \\
\hline $\mathrm{PWV}, \mathrm{m} / \mathrm{s}$ & $8.5 \pm 1.9$ & $8.6 \pm 1.9$ & $8.3 \pm 1.7$ & $<0.0001$ \\
\hline Diabetes, $n(\%)$ & $708(24.5)$ & $533(34)$ & $175(13.2)$ & $<0.0001$ \\
\hline Fasting glucose, mg/dl & $102(53-458)$ & $106(53-458)$ & $99(71-393)$ & $<0.0001$ \\
\hline BMI, $\mathrm{kg} / \mathrm{m}^{2}$ & $28.4 \pm 4.4$ & $28.6 \pm 4.2$ & $28.1 \pm 4.5$ & 0.007 \\
\hline Level of exercise ${ }^{\#}, n(\%)$ & $1334(46.2)$ & $573(36.6)$ & $761(57.6)$ & $<0.0001$ \\
\hline Family history of CAD, $n(\%)$ & $540(18.7)$ & $373(23.8)$ & $167(12.6)$ & $<0.0001$ \\
\hline Heart rate, bpm & $70.4 \pm 12.2$ & $68.8 \pm 12.5$ & $72.3 \pm 11.5$ & $<0.0001$ \\
\hline FRS $<10, n(\%)$ & $1312(45.4)$ & $629(40.2)$ & $683(51.7)$ & * \\
\hline GRS & $0.58 \pm 0.7$ & $0.69 \pm 0.8$ & $0.49 \pm 0.6$ & $<0.0001$ \\
\hline $10 \leq \mathrm{FRS} \leq 20, n(\%)$ & $1049(36.3)$ & $583(37.2)$ & $466(35.2)$ & $*$ \\
\hline GRS & $0.56 \pm 0.6$ & $0.62 \pm 0.7$ & $0.48 \pm 0.4$ & $<0.0001$ \\
\hline FRS $>20, n(\%)$ & $527(18.2)$ & $354(22.6)$ & $173(13.1)$ & $*$ \\
\hline GRS & $0.62 \pm 0.6$ & $0.70 \pm 0.7$ & $0.47 \pm 0.4$ & $<0.0001$ \\
\hline
\end{tabular}

${ }^{\dagger} \mathrm{LDL}>100, \mathrm{HDL}<40$ for men and $<45$ for women, Triglycerides $>150$ and Apo B $>100$; HDL, High-density lipoprotein; LDL, Low-density lipoprotein; 'Current smokers or $<5$ years of cessation; SBP, Systolic blood pressure; DBP, Diastolic blood pressure; PWV, Pulse wave velocity; BMI, Body mass index; \#More than 40min/week; CAD, Coronary Artery Disease; bpm, beat per minute; FRS - 10-year Framingham risk score in \%; *Comparison of the 3 subgroups of FRS, $p<0.0001$; GRS, Genetic risk score; Biochemical variables are presented by median (minimum - maximum) and other continuous variables with mean \pm standard deviation. Statistically significant for $p<0.05$. 
Table 2 - Logistic Regression*model for CAD in subgroups of Framingham 10-year risk $(<10 ; 10-20 \% ;>20 \%)$ and in total population.

\begin{tabular}{|c|c|c|c|c|c|c|c|c|}
\hline \multirow[t]{2}{*}{ Variables } & \multicolumn{2}{|c|}{ FRS $<10 \%$} & \multicolumn{2}{|c|}{ FRS $10 \%-20 \%$} & \multicolumn{2}{|c|}{ FRS $>20 \%$} & \multicolumn{2}{|l|}{ Total } \\
\hline & $\begin{array}{c}\text { OR } \\
(95 \% \mathrm{CI})\end{array}$ & $p$-value & $\begin{array}{c}\text { OR } \\
(95 \% \mathrm{CI})\end{array}$ & $p$-value & $\begin{array}{c}\text { OR } \\
(95 \% \mathrm{CI})\end{array}$ & $p$-value & $\begin{array}{c}\text { OR } \\
(95 \% \mathrm{CI})\end{array}$ & $p$-value \\
\hline Hypertension & $3.25(2.52-4.18)$ & $<0.0001$ & $1.88(1.39-2.56)$ & $<0.0001$ & --- & --- & $2.10(1.77-2.48)$ & $<0.0001$ \\
\hline Diabetes & $2.93(1.90-4.52)$ & $<0.0001$ & $4.34(3.14-6)$ & $<0.0001$ & $3.56(2.34-5.4)$ & $<0.0001$ & $3.19(2.61-3.91)$ & $<0.0001$ \\
\hline Dyslipidemia & $1.71(1.28-2.29)$ & $<0.0001$ & --- & --- & --- & --- & $1.30(1.03-1.65)$ & 0.030 \\
\hline Smoking status & $4.92(3.66-6.62)$ & $<0.0001$ & $3.57(2.65-4.8)$ & $<0.0001$ & $3.11(2.04-4.73)$ & $<0.0001$ & $3.44(2.89-4.1)$ & $<0.0001$ \\
\hline GRS & $1.76(1.41-2.2)$ & $<0.0001$ & $1.86(1.39-2.49)$ & $<0.0001$ & $2.49(1.54-4.05)$ & $<0.0001$ & $1.87(1.58-2.21)$ & $<0.0001$ \\
\hline Constant & 0.16 & $<0.0001$ & 0.24 & $<0.0001$ & 0.342 & $<0.0001$ & 0.21 & $<0.0001$ \\
\hline
\end{tabular}

*Using Forward Wald Conditional Regression method (SPSS v. 19.0). FRS, Framingham risk score; OR, Odds ratio; CI, Confidence interval; GRS, Genetic risk score; Dashed points represent co-variables not significant after adjusted multivariate analysis; Statistically significant for $p<0.05$.

demia $(\mathrm{OR}=1.30 ; 95 \% \mathrm{CI}: 1.03-1.65)$ were independently and significantly associated to CAD risk $(p<0.05)$ (Table 2).

We compared the AUC for the Framingham + GRS model with the basal AUC for the Framingham model, as shown in Figure 1. Framingham yielded an AUC of 0.586 ( $95 \%$ CI: $0.568-0.605$ ), predicting CAD moderately well. The model including the GRS was more discriminative than Framingham alone (AUC $=0.626$; 95\% CI: $0.608-$ 0.644; DeLong test $\mathrm{p}<0.0001)$. The increase in predictive accuracy for the Framingham + GRS model was 4\% (95\% CI: $2.6 \%-5.4 \%)$.
For each subgroup of Framingham, we found that including the GRS to the model with TRF only, the AUC increased significantly (Table 3 ), showing that GRS adds predictive value to TRF in all the 3 risk subgroups. Specifically, in individuals within the low risk subgroup, the AUC was 0.72 for the TRF model and 0.75 for the TRF + GRS model $(p<0.0001)$. The increase in predictive accuracy in this subgroup was $2.5 \%$ (95\% CI: $1.4-3.6 \%)$. This difference was small, but significant (DeLong test for correlated ROC curves $p<0.0001)$. Similarly, in individuals within intermediate FRS subgroup, the AUC was 0.70 for the TRF model and 0.73 for the TRF + GRS model. The in-

Table 3 - Reclassification table comparing predicted CAD risk with and without GRS.

\begin{tabular}{|c|c|c|c|c|}
\hline & \multirow[b]{2}{*}{$\begin{array}{c}\text { Total } \\
(\mathrm{n}=2888)\end{array}$} & \multicolumn{3}{|c|}{ FRS } \\
\hline & & $\begin{array}{c}<10 \% \\
(\mathrm{n}=1312)\end{array}$ & $\begin{array}{c}10-20 \% \\
(\mathrm{n}=1049)\end{array}$ & $\begin{array}{c}>20 \% \\
(\mathrm{n}=527)\end{array}$ \\
\hline CAD patients (n) & 1566 & 629 & 583 & 354 \\
\hline Controls (n) & 1322 & 683 & 466 & 173 \\
\hline NRI (\%) & 31.7 & 32.3 & 30.4 & 29.8 \\
\hline$(25.0-38.4)$ & $(22.4-42.3)$ & $(19.0-41.8)$ & $(13.1-46.6)$ & \\
\hline$(95 \% \mathrm{CI})$ & $p<0.0001$ & $p<0.0001$ & $p<0.0001$ & $p=0.0005$ \\
\hline IDI (\%) & 2.3 & 2.4 & 2.1 & 3.3 \\
\hline \multirow[t]{2}{*}{$(95 \% \mathrm{CI})$} & $(1.8-2.9)$ & $(1.6-3.2)$ & $(1.3-2.9)$ & $(1.9-4.7)$ \\
\hline & $p<0.0001$ & $p<0.0001$ & $p<0.0001$ & $p<0.0001$ \\
\hline \multicolumn{5}{|l|}{ AUC } \\
\hline TRF & 0.716 & 0.724 & 0.701 & 0.676 \\
\hline$(95 \% \mathrm{CI})$ & $(0.699-0.732)$ & $(0.699-0.748)$ & $(0.672-0.728)$ & $(0.634-0.716)$ \\
\hline $\mathrm{TRF}+\mathrm{GRS}$ & 0.741 & 0.749 & 0.726 & 0.720 \\
\hline$(95 \% \mathrm{CI})$ & $(0.724-0.757)$ & $(0.725-0.772)$ & $(0.698-0.753)$ & $(0.680-0.758)$ \\
\hline$p$-value for the difference & $<0.0001$ & 0.0001 & 0.0002 & 0.003 \\
\hline \multicolumn{5}{|l|}{ Nagelkerke R Square } \\
\hline TRF & 0.190 & 0.213 & 0.166 & 0.138 \\
\hline TRF+GRS & 0.219 & 0.242 & 0.191 & 0.184 \\
\hline
\end{tabular}

FRS, 10-year Framingham risk score; CAD, Coronary Artery Disease; NRI, Net Reclassification Improvement continuous NRI); CI, Confidence Interval; IDI, Integrated Discrimination Improvement; AUC, Area Under the receiver operating characteristic Curve; TRF, Traditional Risk Factors (Dyslipidemia, Smoking, Diabetes and Hypertension); GRS, Genetic risk score. 
crease in the predictive accuracy for the AUC model in this subgroup was $2.6 \%(95 \%$ CI: $1.2-3.9 \%)$ at a significant $p$ level $(p=0.0002)$. Finally, a surprising significant (DeLong's test for correlated ROC curves $\mathrm{p}=0.003$ ) addition to the model's predictive accuracy was seen in the higher risk of FRS group with an AUC increasing from 0.68 to 0.72 . The increase in predictive accuracy for the AUC model (TRF + GRS model) in this group was 4.4\% ( $95 \%$ CI: $1.5-7.3 \%$ ). For all subgroups, the $95 \% \mathrm{CI}$ of the increase did not include zero, suggesting that the increase in AUC caused by the GRS inclusion is statistically significant. The addition of GRS to TRF improved the risk classification of the models. The new marker provided a continuous NRI of $32.3 \%$ (95\%CI: $22.4-42.3 \%$; $p<0.0001)$ in the low risk group; $30.4 \%$ (95\%CI: $19.0-41.8 \% ; p<0.0001)$ in the intermediate FRS group and $29.8 \% \quad(95 \% \mathrm{CI}$ : $13.1-46.6 \% ; p=0.0005$ ) in the high risk subgroup (Table 3). Furthermore, the inclusion of GRS to TRF also provided an IDI of $2.4 \%$ (95\%CI: $1.6-3.2 \% ; p<0.0001)$ in the low risk group; $2.1 \%$ (95\%CI: $1.3-2.9 \% ; p<0.0001)$ in the intermediate risk group and 3.3\% (95\%CI: $1.9-4.7 \% ; p<0.0001)$ in the high-risk group. Full reclassification results are provided in Table 3.

The Nagelkerke $\mathrm{R}$ square value increased in each FRS subgroup with inclusion of GRS, that is, this new variable improved the proportion of variation explained in the model. Therefore, it can be stated that the disease is explained at $19.1 \%$ by the independent variables in the intermediate risk group. The values presented in Table 3 confirm that the selected variables present a significant explanatory degree about the dependent variable.

A complementary study of categorical NRI is shown in Supplementary Table S2. In the Framingham low risk subgroup $(<10 \%)$, a $27.5 \%$ NRI was found in the control population and a $-7.8 \% \mathrm{NRI}$ in CAD subgroup. In intermediate Framingham risk subgroup (10-20\%), the opposite result was found with $18 \%$ of CAD patients being reclassified into higher risk whereas $0.6 \%$ of controls into lower risk. Finally, for the higher risk subgroup ( $>20 \%$ ), both patients and controls were reclassified into higher risk groups (5.9\% and $-1.7 \%$, respectively). Overall reclassification for each Framingham subgroup (case- control) was positive and expressed as a categorical NRI of $19.7 \%, 18.7 \%$ and $4.2 \%$, respectively.

\section{Discussion}

We have downgraded our expectations after an unfulfilled enthusiastic time of expecting genes to account for hidden heritability in $\mathrm{CAD}$, not meant for generalized use in the actual guidelines for cardiovascular prevention. For $1-5 \%$ of allelic frequencies and ORs for CAD/MI at GWAS level from 1.1- 1.5 reported in the literature will produce moderate risk improvement if added to standard risk stratification models, such as Framingham (Morrison et al., 2007; Van der Net et al., 2009). These models have reason- able to good risk prediction curves, as modeled with Cstatistics ranging from $68-72 \%$ (Humphries et al., 2008; Paynter et al., 2010). Nevertheless improved accuracy is expected when gene-gene inter-mapping and the impact of some epigenetic factors are accounted for in CAD risk.

The current case-control study assesses the contribution of genetic risk for CAD in a southern European population controlled for age and sex. In the multivariate model, GRS presents a cardiovascular risk independent of the risk conferred by TRF. Thus, it can improve the predictive capacity of TRF scores. According to the results, standard risk Framingham evaluation states good AUC levels across all risk subgroups, with an AUC ranging from 0.676 to 0.749. Apart from its mathematical calculation, the intermediate risk subgroup represents the vast majority of patients followed at the outpatient clinics. The low-age patient with one or two risk factors and low calculated cardiovascular risk is frequently left out of intensive prevention, such as statin introduction, active measures for tobacco eviction, or inclusion in exercise program.

Here, we report that a GRS based on 32 CAD risk alleles, identified previously in GWAS databases, independently and significantly predicts $\mathrm{CAD}$ with an increased risk of $80 \%$ (1.8 times). Our observations line up with several reports already addressing the impact of adding GRS to standard risk stratification. Recently, Iribarren et al. (2016) have reported, in a large scale cohort living in the US, improved predictive capacity and discrimination index for four GRS starting from just 8 or 12 and up to 36 to 51 genetic variants. Likewise Tada et al. (2016) also reported that in a panel of 23,595 participants followed-up for 14 years, two GRS of 27 and 50 SNPs improved all measures of discrimination and reclassification.

Despite the modest predictive ability, our genetic risk model seems useful in preventive health care and disease prevention to correctly identify individuals at intermediate and high-risk groups. We observed a general tendency for both measures of reclassification improvement, the NRI and IDI, to increase after addition of the GRS to the basic risk function. The continuous NRI was $30.8 \%$ for the entire cohort (95\% CI: 24.0\%-37.5\%). However, reclassification improvement was more noticeable in the intermediate risk group, where it was statistically significant (NRI: $33.7 \%$, $95 \%$ CI $25.6 \%-41.8 \%$; IDI: $1.8 \%, 95 \%$ CI $1.2 \%-2.3 \%$ ). It is important to note that the constructed reclassification tables follow clinically relevant strata of 5 , and 20 percent 10 -year risk, in order to have direct clinical application. But limitations in the interpretation of data apply, because the prevalence of CAD in any patient study population is much higher than in a normal population, and thus, frequencies and risks are highly overestimated. The feasibility and clinical application of the GRS approach strategy will depend not only on the predictive capacity of the risk model, but also on the threshold level that is chosen. 
We believe that genetic information will improve preventive strategies targeting individuals at very high genetic risk even though this may be a small subgroup. Nevertheless, an issue that deserves remarks relates to genetic information, adverse events, and even cardiovascular mortality. It is irrefutable that GRS is associated with worse prognosis and outcomes. Several GRS, including SNPs associated with CHD, have been associated with incident events in distinct cohorts (CARDIoGRAMplusC4d Consortium, 2013).

These studies have been conducted in both primary and secondary prevention subpopulations. Long-term prospective cohorts have validated GRS as a marker for incident events across all risk categories in primary care (Voight et al., 2012; Krarup et al., 2015). Mega et al. (2015) have also analyzed GRS orientated therapy with statins reporting interesting number needed to treat reaching just 20 to 25 patients with high GRS to avoid one incident adverse outcome. Also, Voight et al. (2012) have already tested GRS in a Mendelian randomization design trial, and thus, GRS has been pointed out as an effective tool for early statin introduction in patients with a concordant genotypic score and phenotypic LDL elevation (Voight et al., 2012). Similarly, in a secondary prevention setting, our group has reported, in 1555 patients with documented CAD, significantly higher cardiovascular mortality in patients with higher GRS (Pereira et al., 2017).

Considerably disparate numbers of gene variants and different population settings have been validated in these trials, ranging from 13 to a few hundred genes. Not in all of these studies with positive association with adverse outcomes at the long-term follow-up, the NRI was statistically significant (Ripatti et al., 2010). But this was not a justification for its clinical uses (Pencina et al., 2009). Furthermore, Pencina et al. (2011) have already addressed this limitation of comparing NRI, stating that continuous NRI may not be adequate in case-control studies. Similarly, distinct risk cut-offs and the number of risk categories in categorical NRI make inter-study comparisons rather difficult. Nevertheless, and apart from the limitation of using NRI to improve models that already perform well (Pencina et al., 2011), in this study, both continuous and categorical metric formulas can be of sufficient discrimination power to be useful in clinical settings, especially regarding the intermediate risk group, where classical and standard risk stratification seems to be open to sampling errors.

Irrespective of their role in preventive strategy control and relative mortality reduction, classical risk factors can change over time with lifestyle and drug interventions. Also, many biochemical and phenotypic biomarkers, like hs-CRP and PWV, are powerful predictors of disease risk for short-term concern, but less accurate in assessing lifetime risk. Screening for risk stratification is an important issue. Genetic information is an attractive measure for risk prediction with a number of advantages over classical risk stratification. Though its true predictive power in different clinical scenarios can be quite disparate, by being selftailored and highly stable over time its use may gain preference over classical measures. Furthermore, as It is expected that financial cost related to genome sequencing will further decline in the near future and, already in short-term, additional genetic prediction will become available as regular biomarker determinations and, in this way, can reach larger population coverage. Our findings confirm the importance of genetic contributions to CAD disease even when adjusting for traditional risk stratification.

\section{Strengths and limitations of the study}

A significant strength was the use of a genetically isolated population. This has been especially valuable for mapping rare recessive disorders, but many researchers believe that this could be a solution for more complex disorders as well, because of the relatively uniform genetic background of the population. Some culturally and genetically isolated populations have a more similar lifestyle and share eating habits and a natural environment, thus reducing environmental variation. Often, these populations have been founded by a small number of individuals, followed by a period of genetic isolation, during which genetic drift might have been seen, and population expansion mainly occurred by population growth and not by immigration (Jorde and Wooding, 2004). Another strength of this study is the use of a gold standard of angiography for CAD phenotype assignment, which refines the group of interest.

Our study has included a limited number of genetic variants associated with CAD. Although there is no established minimal number of genes to include in a genetic score, and this number may depend on allele frequencies specific to the population under analysis, the inclusion of more SNPs into our score could increase the predictive power for $\mathrm{CAD}$ and other outcomes. Further studies evaluating the clinical utility of adding a GRS in very large samples of individuals are underway.

Continuous and categorical NRI may have improved our prediction performance for each category risk. Nevertheless, as previously stated, critical issues about the limitation of using NRI, either as a continuous or categorical variable, are still under debate and not conclusively solved. In clinical settings, additional stratification strategies for $\mathrm{CAD}$, such as biomarkers, imaging modalities, or genetic profiles, will have to be calculated individually or for the intermediate risk group.

\section{Conclusions}

Overall, our findings demonstrate that GRS is independent of TRF in a single-center cohort with adequate population stratification. GRS improved the predictive risk of FRS across all subgroups in 10-year risk estimates according to the Framingham risk prediction function. A marginal increase in reclassifications measures was found, with 
a similar pattern across all subgroups. Further debate is warranted about the incorporation of genetic data in subgroups known to be subestimated with respect to standard stratification.

\section{Acknowledgments}

We are very grateful to Elsa Sousa who handled all the administrative procedures, and to Rita Freitas (CIDEHUS, Évora University, Portugal) who reviewed the manuscript with respect to statistical analysis.

\section{References}

Abdi H and Williams LJ (2010) Principal component analysis. WIREs Comp Stat 2:433-459.

Asmar R, Benetos A, Topouchian J, Laurent P, Pannier B, Brisac AM, Target R and Levy BI (1995) Assessment of arterial distensibility by automatic pulse wave velocity measurement: Validation and clinical application studies. Hypertension 26:485-490.

CARDIoGRAMplusC4d Consortium (2013) Large-scale association analysis identifies new risk loci for coronary artery disease. Nat Genet 45:25-33.

Chan L and Boerwinkle E (1994) Gene-environment interactions and gene therapy in atherosclerosis. Cardiol Rev 2:130-137.

Chobanian AV, Bakris GL, Black HR, Cushman WC, Green LA, Izzo Jr JL, Jones DW, Materson BJ, Oparil S and Wright Jr JT (2003) The seventh report of the Joint National Committee on Prevention, Detection, Evaluation and Treatment of High Blood Pressure: The JNC 7 report. JAMA 289:25602572.

Coronary Artery Disease Genetics Consortium (2011) A genome-wide association study in Europeans and South Asians identifies five new loci for coronary artery disease. Nat Genet 43: 339-344.

DeLong ER, DeLong DM and Clarke-Pearson DL (1988) Comparing the areas under two or more correlated receiver operating characteristic curves: A nonparametric approach. Biometrics 44: 837-845.

Ganna A, Magnusson PKE, Pedersen NL, de Faire U, Reilly M, Arnlöv J, Sundström J, Hamsten A and Ingelsson E (2013) Multilocus genetic risk score for coronary heart disease prediction. Arterioscler Thromb Vasc Biol 33:2267-2272.

Expert Panel on Detection Evaluation and Treatment of High Blood Cholesterol in Adults (2001) Executive summary of the third report of the National Cholesterol Education Program (NCEPT) Expert Panel on Detection Evaluation and Treatment of High Blood Cholesterol in Adults (Adult Treatment Panel III). JAMA 285:2486-249.

Humphries SE, Yiannakouris N and Talmud PJ (2008) Cardiovascular disease risk prediction using genetic information (gene scores): Is it really informative? Curr Opin Lipidol 19:128-132.

Iribarren C, Lu M, Jorgeson E, Martinez M, Lluis-Ganella C, Subrirana I, Salas E and Elosua R (2016) Clinical utility of multi-marker genetic risk scores for prediction of incident coronary heart disease: A cohort study among over 51 thousand Individuals of European ancestry. Circ Cardiovasc Genet 9:531-540.
Genuth S, Alberti KG, Bennett P, Buse J, Defronzo R, Kahn R, KItzmiller J, Knowler WC, Lebovitz H, Lernmark A et al. (2003) Follow-up report on the diagnosis of diabetes mellitus.Diabetes Care 26:3160-3167.

Jorde LB and Wooding SP (2004) Genetic variation, classification and 'race'. Nat Genet 3611 Suppl:S28-S33.

Krarup NT, Borglykke A, Allin KH, Sandholt CH, Justesen JM, Andersson EA, Grarup N, Jørgensen T, Pedersen $\mathrm{O}$ and Hansen T (2015) A genetic risk score of 45 coronary artery disease risk variants associates with increased risk of myocardial infarction in 6041 Danish individuals. Atherosclerosis 240:305-310.

Lloyd-Jones DM, Nam BH, D'Agostino RB, Levy D, Murabito JM, Wang TJ, Wilson PW and O'Donnell CJ (2004) Parental cardiovascular disease as a risk factor for cardiovascular disease in middle-aged adults: A prospective study of parents and offspring. JAMA 291:2204-2211.

Marenberg ME, Risch N, Berkman LF, Floderus B and de Faire U (1994) Genetic susceptibility to death from coronary heart disease in a study of twins. N Engl J Med 330:1041-1046.

Mega JL, Stitziel NO, Smith JG, Chasman DI, Caulfield MJ, Devlin JJ, Nordio F, Hyde CL, Cannon CP and Sacks FM (2015) Genetic risk, coronary heart disease events, and the clinical benefit of statin therapy: An analysis of primary and secondary prevention trials. Lancet 385:2280-2287.

Mons U, Müezzinler A, Gellert C, Schöttker B, Abnet CC, Bobak M, de Groot L, Freedman ND, Jansen E, Kee F et al. (2015) Impact of smoking and smoking cessation on cardiovascular events and mortality among older adults: Meta-analysis of individual participant data from prospective cohort studies of the CHANCES consortium. BMG 350:h1551.

Morrison AC, Bare LA, Chambless LE, Ellis SG, Malloy M, Kane JP, Pankow JS, Devlin JJ, Willerson JT and Boerwinkle E (2007) Prediction of coronary heart disease risk using a genetic risk score: The Atherosclerosis Risk in Communities Study. Am J Epidemiol 166:28-35.

Murabito JM, Pencina MJ, Nam BH, D'Agostino RB, Wang TJ, Lloyd-Jones D, Wilson PW and O'Donnell CJ (2005) Sibling cardiovascular disease as a risk factor for cardiovascular disease in middle-aged adults. JAMA 294:3117-3123.

National Institutes of Health; National Heart, Lung, and Blood Institute; NHLBI Obesity Education Initiative; North American Association for the Study of Obesity (2000) The Practical Guide: Identification, Evaluation, and Treatment of Overweight and Obesity in Adults. National Institutes of Health; National Heart, Lung, and Blood Institute; NHLBI Obesity Education Initiative; North American Association for the Study of Obesity, Bethesda.

Paynter NP, Chasman DI, Pare G, Buring JE, Cook NR, Miletich JP and Ridker PM (2010) Association between a literature-based genetic risk score and cardiovascular events in women. JAMA 303:631-637.

Pencina MJ, D'Agostino RB, Larson MG, Massaro JM, Vasan RS (2009) Predicting the 30-year risk of cardiovascular disease: The Framingham heart study. Circulation 119:3078-3084.

Pencina MJ, Steverberg EW and D'Agostino RB Sr (2011) Extensions of net reclassification improvement calculations to measure usefulness of new biomarkers. Stat Med 30:11-21.

Pereira A, Mendonça MI, Sousa AC, Borges S, Freitas S, Henriques E, Rodrigues M, Freitas AI, Guerra G, Ornelas I et al. (2017) Genetic risk score and cardiovascular mortality 
in a Southern European population with coronary artery disease. Int J Clin Pract 71: e12956.

Ridker PM, Buring JE, Rifai N and Cook NR (2007) Development and validation of improved algorithms for the assessment of global cardiovascular risk in women: The Reynolds Risk Score. JAMA 297:611-619. - não foi citada no corpo do texto

Ridker PM, Paynter NP, Rifai N, Gaziano JM and Cook NR (2008) C-reactive protein and parental history improve global cardiovascular risk prediction: The Reynolds Risk Score for men. Circulation 118:2243-2251.

Ripatti S, Tikkanen E, Orho-Melander M, Havulinna AS, Silander K, Sharma A, Guiducci C, Perola M, Jula A, Sinisalo J et al. (2010) A multilocus genetic risk score for coronary heart disease: Case control and prospective cohort analyses. Lancet 376:1393-1400.

Schunkert H, König IR, Kathiresan S, Reilly MP, Assimes TL, Holm H, Preuss M, Stewart AF, Barbalic M and Gieger C (2011) Large-scale association analysis identifies 13 new susceptibility loci for coronary artery disease. Nat Genet 43:333-338.

Tada H, Melander O, Louie JZ, Catanese JJ, Rowland CM, Devlin JJ, Kathiresan S, Shiffman D (2016) Risk prediction by genetic risk scores for coronary heart disease is independent of self-reported family history. Eur Heart J 37:561-567.

Van der Net JB, Janssens AC, Defesche JC, Kastelein JJ, Sijbrands EJ and Steyerberg EW (2009) Usefulness of ge- netic polymorphisms and conventional risk factors to predict coronary heart disease in patients with familial hypercholesterolemia. Am J Cardiol 103:375-380.

Voight BF, Peloso GM, Orho-Melander M, Frikke-Schmidt R, Barbalic M, Jensen MK, Hindy G, Hólm H, Ding EL and Johnson T (2012) Plasma HDL cholesterol and risk of myocardial infarction: A Mendelian randomisation study. Lancet 380:572-580.

Welter D, MacArthur J, Morales J, Burdett T, Hall P, Junkins H, Klemm A, Flicek P, Manolio T and Hindorff L (2014) The NHGRI GWAS Catalog, a curated resource of SNP- trait associations. Nucleic Acids Res 42:D1001-D1006.

\section{Supplementary material}

The following online material is available for this article:

Table S1 - List of the 32 genetic variants previously associated with CAD risk, selected to create the GRS in our Population.

Table S2 - Reclassification table comparing predicted CAD risk with and without GRS.

Associate Editor: Angela M. Vianna-Morgante

License information: This is an open-access article distributed under the terms of the Creative Commons Attribution License (type CC-BY), which permits unrestricted use, distribution and reproduction in any medium, provided the original article is properly cited. 